\title{
The Minimum Sum Representation as an Index of Voting Power
}

\author{
Josep Freixas* $^{*}$ and Serguei Kaniovski ${ }^{\dagger}$
}

July 17,2013

\begin{abstract}
We propose a new power index based on the minimum sum representation (MSR) of a weighted voting game. The MSR offers a redesign of a voting game, such that voting power as measured by the MSR index becomes proportional to voting weight. The MSR index is a coherent measure of power that is ordinally equivalent to the Banzhaf, Shapley-Shubik and Johnston indices. We provide a characterization for a bicameral meet as a weighted game or a complete game, and show that the MSR index is immune to the bicameral meet paradox. We discuss the computation of the MSR index using a linear integer program and the inverse MSR problem of designing a weighted voting game with a given distribution of power.
\end{abstract}

Keywords: minimum integer sum representation; power indices; bicameral meet; rankings; proportional design between shares and power

\section{Introduction}

Consider a simple majority voting game, in which two voters have 49 votes each, whereas a third voter has 2 votes only. Let the weights of the voters represent their contributions to a common cause, or ownership stakes in a joint equity arrangement. Examples of the latter type include shareholder voting in corporations and country member voting in the multilateral institutions of the Bretton Woods Accord. Simple majority rule stipulates that, in order for a coalition to win, it must command at least 51 votes.

The voters have glossily unequal weights and yet are equally powerful because a coalition of any two of them wins. Why would the larger voters contribute too much, relative to the power they receive? Assuming integer weights and holding the voting weight of the smallest voter fixed at 2 , the larger voters would have an incentive to reduce their voting weights to 2 . This is the minimal contribution that preserves an equal distribution of power after lowering the quota to

*Departament de Matemàtica Aplicada 3 i Escola Politècnica Superior d'Enginyeria de Manresa, Universitat Politècnica de Catalunya. Research partially supported by Grants SGR 2009-1029 of "Generalitat de Catalunya" and MTM2012-34426/FEDER "del Ministerio de Economía y Competitividad".

E-mail: josep.freixas@upc.edu.

${ }^{\dagger}$ Austrian Institute of Economic Research (WIFO), P.O. Box 91, A-1103 Vienna, Austria. E-mail: serguei.kaniovski@wifo.ac.at. 
4. The sum of voting weights, or the joint stock, shrinks from 100 to 6 . In fact, we may expect a race to the bottom until the weights fall to 1 (quota 2 , joint stock 3 ). ${ }^{1}$

We started from the game $[51 ; 49,49,2]$ and ended with the game $[2 ; 1,1,1]$. This is the minimum sum representation (MSR) of a game with three equally powerful voters. It turns out that the share in the sum of voting weights of an MSR is a valid measure of power. We call this new measure the 'MSR index'. In the above games, the power vector reads $(1 / 3,1 / 3,1 / 3)$. Powers according to our new measure are thus proportional to voting weights in the MSR.

We show that the MSR index is a coherent measure of power. According to Freixas and Gambarelli (1997), a measure is coherent if it: vanishes for powerless players, is invariant under isomorphisms, leads to the measured power being shared among the voters, and is strictly monotonic. The monotonicity is based on Isbell's (1956) desirability relationship (see also, Taylor and Zwicker 1999). In weighted voting games, strict Isbell monotonicity implies strict monotonicity of power in voting weight. But Isbell monotonicity also applies to more general types of complete simple games for which the desirability relationship is total. Weighed voting games are a class of complete simple games. For weighted voting games, the Freixas and Gambarelli coherency criteria are equivalent to the 'minimal adequacy postulate' by Felsenthal and Machover (1998) (p.222), plus the dominance criterion (Ibid., ch.7.6), which they find sufficiently important to disqualify two existing power indices that violate it (Deegan and Packel (1978) and Holler (1982)).

It is important to emphasize that the domain of application of the MSR index is confined to weighted voting games. In this respect the MSR index is less general than the existing power indices that can be computed for any simple voting game. It is, however, weighted voting games that are relevant to the applied power measurement and institutional design. The MSR index is ordinally equivalent to the Banzhaf, Shapley-Shubik and Johnston indices, so that all three indices produce the same power ranking in any weighted voting game.

Further exploring the properties of the MSR index, we show that it is immune to the bicameral meet paradox. A bicameral meet is a union of two voting games. Respecting bicameral meet requires that the merging of two voting games not change the relative powers of voters, who were in the same game prior to the merge. The quirk is that a bicameral meet of two complete games may not be complete, and a bicameral meet of two weighted games may not be weighted. We provide a characterization of simple voting games, in which these two properties carry over from the constituent games to the union game.

The paper is structured as follows. Sections 2 and 3 recapitulate the theoretical foundations of simple voting games and power indices, respectively. Sections 4 and 5 discuss the MSR, formulate the MSR index and establish its coherency as a measure of power. The index is uniquely determined and can be computed using an integer linear program. The inverse MSR problem of designing a weighted voting game with a given distribution of power can be solved with the same method used to compute the index. This stands in contrast to the existing power indices, whose inverse problems are significantly more difficult than direct computations. Section 6 illustrates the MSR index on two constituencies of the IMF and the German Bundestag after the general election of 2009. Vulnerabilities to certain anomalies, commonly referred to as voting paradoxes, are discussed in Section 7. In this section, we obtain the characterization for bicameral meet games and use it to show that the MSR index respects the bicameral meet

\footnotetext{
${ }^{1}$ In a non-cooperative meta-game, in which players' strategies are their voting weights and their payoff is voting power, this is unlikely to be a stable equilibrium because each player would benefit from unilaterally increasing her voting weight.
} 
postulate. In a symmetric weighted voting game, the power of a voting bloc according to the MSR index equals the sum of individual powers of its members. The MSR index is thus neutral with respect to aggregating powers in symmetric weighted voting games. This is different from the Banzhaf and Shapley-Shubik indices, which can assign more or less than the sum of individual powers to the bloc. Since a symmetric weighted voting game with a voting bloc is a particular case of a game with an a priori union, we provide a definition of the MSR index for games with a priori unions and discuss the computation of the MSR index in such games. The last section offers concluding remarks.

\section{Simple Voting Games}

A simple voting game (SVG) is a collection $\mathcal{W}$ of sets contained in the finite set $N=\{1,2, \ldots, n\}$, satisfying the following properties:

(i). $N \in \mathcal{W}$;

(ii). $\emptyset \notin \mathcal{W}$;

(iii). Whenever $S \subseteq T \subseteq N$ and $S \in \mathcal{W}$, then also $T \in \mathcal{W}$;

We shall refer to $N$ as the assembly of $\mathcal{W}$. The members of $N$ are the voters in $\mathcal{W}$. A set of voters, a subset of $N$, is called a coalition. The cardinal of a set of voters $S$, or the number of voters in coalition $S$, is denoted by $|S|$.

Any member of $\mathcal{W}$ is a winning coalition. If $S \subseteq N$ but $S \notin \mathcal{W}$, then $S$ is a losing coalition. A winning coalition is minimal if it has no proper winning subset. The set of winning coalitions $\mathcal{W}$, or the set of minimal winning coalitions $\mathcal{W}^{m}$, completely characterizes the SVG.

A voter $i$ is a vetoer if $i \in S$ for all $S \in \mathcal{W}^{m}$. A voter $i$ is null if $i \notin S$ for every $S \in \mathcal{W}^{m}$. A vetoer $i$ is a dictator if $\mathcal{W}^{m}=\{\{i\}\}$. In this case, all players in $N \backslash\{i\}$ are null. A voter $i$ in an SVG is called trivial if she is either a vetoer, or a null voter. A simple voting game comprising trivial voters only is called a unanimity of a coalition game, and has a singleton $\mathcal{W}^{m}=\{T\}$ for some $\emptyset \subsetneq T \subseteq N$ as the set of minimal winning coalitions.

An SVG is a weighted voting game (WSVG) if one can assign to each $i \in N$ a nonnegative real number $w_{i}$, and fix a real positive number $q$, such that

$$
\mathcal{W}=\{S \subseteq N: w(S) \geq q\}, \text { where } w(S)=\sum_{i \in S} w_{i}
$$

Here, $w_{i}$ is the voting weight of voter $i$, and $q$ is the number of affirmative votes required for a decision to be passed. A representation of a WSVG with a quota $q$ and weights $w_{i}$ for every $i \in N$ is denoted by $\left[q ; w_{1}, w_{2}, \ldots, w_{n}\right]$, where $n=|N|$ is the number of voters. Should the vector of weights $w=\left(w_{1}, w_{2}, \ldots, w_{n}\right)$ be specified, we may use the shorter notation $[q ; w]$ instead.

Two distinct representations of a WSVG are equivalent if they induce the same set $\mathcal{W}$. For example, $[51 ; 49,49,2] \equiv[102 ; 98,98,4]$, since $\mathcal{W}=\{\{1,2\},\{1,3\},\{2,3\},\{1,2,3\}\}$ in both games. This shows that the number of WSVG equivalent to a given WSVG is infinite, as rescaling the weights and quota by the same factor preserves the set of winning coalitions. 


\section{Coherent Power Measures}

A power index is a mapping $K$ that assigns to each SVG a vector in $\mathbb{R}_{+}^{n}$. A power index on a subclass of SVGs, say $\mathcal{S}$, is a mapping $K$ that assigns to each game in $\mathcal{S}$ a vector in $\mathbb{R}_{+}^{n}$. For the purposes of this paper, we consider power indices on the class of WSVGs only.

In addition to nonnegativity, Freixas and Gambarelli (1997) state the additional properties any reasonable measure of power must fulfill as follows:

(i). Null voter: If $i$ is a null voter in $(N, \mathcal{W})$, then $K_{i}(\mathcal{W})=0$;

(ii). Efficiency: $\sum_{i \in N} K_{i}(\mathcal{W})=1$;

(iii). Invariance: $K_{i}(\mathcal{W})=K_{\pi(i)}(\mathcal{W})$ for every bijective map (isomorphism) $\pi: N \rightarrow N$, such that $S \in \mathcal{W} \Leftrightarrow \pi(S) \in \mathcal{W}$;

(iv). Strong monotonicity: if $i \succ_{D} j$ in $(N, \mathcal{W})$ then $K_{i}(\mathcal{W})>K_{j}(\mathcal{W})$.

The null voter property requires the index to vanish for powerless voters. Efficiency requires voting powers to sum to unity. This normalization is appropriate when power justifies a claim on a prize to be shared among the voters (P-power in Felsenthal and Machover (2004)). The more powerful the voter, the larger the share she receives. Null voter and efficiency together imply that a dictator receives the entire prize. Invariance says that any transformation that preserves the set of winning coalitions must also preserve the distribution of power. A rescaling of quota and weights in a weighted voting game should leave the distribution of power unchanged.

Monotonicity is formulated in terms of Isbell's desirability relation. The notation $\succsim_{D}$ denotes a relation on $N$, such that $i \succsim_{D} j$, if $S \cup\{j\} \in \mathcal{W}$ implies $S \cup\{i\} \in \mathcal{W}$ for every $S \subseteq N \backslash\{i, j\}$. Roughly speaking, adding voter $i$ instead of voter $j$ to any coalition $S$ will have the same or better effect on its decisiveness, making $i$ a more desirable addition for the voters comprising $S$.

The game $(N, \mathcal{W})$ is called complete $(\mathrm{CSVG})$ if $\succsim_{D}$ is a total (weak) order. Then:

$$
\begin{aligned}
& i \succ_{D} j \text { if } i \succsim_{D} j \text { but not } j \succsim_{D} i ; \\
& i \approx_{D} j \text { if } i \succsim_{D} j \text { and } j \succsim_{D} i .
\end{aligned}
$$

All WSVGs are CSVGs because $w_{i} \geq w_{j}$ implies $i \succsim_{D} j$. The class of complete simple games is thus more general than the class of weighed voting games. For $n \geq 6$ there exist complete SVGs that are not WSVGs and for $n \geq 4$ there exist SVGs that are not CSVGs.

Taylor and Pacelli (2008) offer a test of completeness. A simple game is complete if it is swap robust, or if a one-for-one exchange of players between any two winning coalitions $S$ and $T$ leaves at least one of the two coalitions winning. One of the players in the swap must belong to $S$ but not $T$, and the other must belong to $T$ but not $S$.

For weighted voting games, the above coherency criteria are equivalent to 'minimal adequacy postulates' for WSVGs by Felsenthal and Machover (1998), plus the strong dominance (Ibid., def.7.1.1 and ch.7.6). The Deegan-Packel index, the Holler index and the Nucleolus violate strong monotonicity, although the Nucleolus $\eta$ cannot reverse the order of the Isbell's desirability relation, i.e., $i \succ_{D} j$ and $\eta_{j}<\eta_{i}$ are incompatible. The Banzhaf, Shapley-Shubik or Johnston indices satisfy strong monotonicity. These three indices are ordinally equivalent in a large class of games that contains all CSVGs (Freixas, Marciniak and Pons 2012). 


\section{The Minimum Sum Representation of a WSVG}

It is well-known that any WSVG admits a representation with nonnegative integer weights and positive integer quota. Let $\mathcal{I}(N, \mathcal{W})$ denote the unbounded set of integer representations for $(N, \mathcal{W})$. If $[q ; w] \in \mathcal{I}(N, \mathcal{W})$, then $[t q ; t w] \in \mathcal{I}(N, \mathcal{W})$ for any positive integer $t$. Geometrically, the set $\mathcal{I}(N, \mathcal{W})$ is a discrete cone of integers in the $(n+1)$-dimensional set $\mathbb{N}^{n+1}$.

A representation $[q ; w]$ of a WSVG $(N, \mathcal{W})$ is called minimum if $w^{\prime} \geq w$, i.e., $w_{i}^{\prime} \geq w_{i}$ for all $i=1,2, \ldots, n$, for all representations $\left[q^{\prime} ; w^{\prime}\right] \in \mathcal{I}(N, \mathcal{W})$. Let $M \mathcal{I}$ be the set of minimum (integer) representations.

A representation $[q ; w]$ of a $\operatorname{WSVG}(N, \mathcal{W})$ has minimum sum if $w^{\prime}(N) \geq w(N)$ for all $\left[q^{\prime} ; w^{\prime}\right] \in \mathcal{I}(N, \mathcal{W})$. Let $s M \mathcal{I}$ be the set of all minimum sum (integer) representations.

Henceforth, we omit the qualification 'integer', as we consider integer-weighted games only, and use the shorter notation $M \mathcal{I}$ and $s M \mathcal{I}$ instead of $M \mathcal{I}(N, \mathcal{W})$ and $s M \mathcal{I}(N, \mathcal{W})$ whenever there is no ambiguity about the game.

Some properties of minimum integer and minimum sum representations have been obtained in Freixas and Molinero (2009) and Freixas and Molinero (2010b).

Proposition 4.1 Let $(N, \mathcal{W})$ be a $W S V G$, and $M \mathcal{I}$ and $s M \mathcal{I}$ be the sets defined above.

(i). MI has at most one element;

(ii). sMI is never empty;

(iii). $M \mathcal{I} \subseteq s M \mathcal{I}$, where the equality arises, if and only if $M \mathcal{I}$ is a singleton.

Let $[q ; w] \in s M \mathcal{I}$ be a representation for the $W S V G(N, \mathcal{W})$, then:

(iv). $q=a$ and $q=b+1$, where $a=\min _{S \in \mathcal{W}} w(S)$ and $b=\max _{S \notin \mathcal{W}} w(S)$;

$(v)$.

$$
\begin{aligned}
& w_{i}=0, \text { if and only if } i \text { is a null voter; } \\
& w_{i}=v, \text { if } i \text { has veto, where } v=w(N)-q+1 ;
\end{aligned}
$$

(vi). The greatest common divisor of $\left\{w_{1}, \ldots, w_{k}\right\}=1$, where $\{1, \ldots, k\}$ denotes the set of non-null voters in $N$.

Freixas and Molinero (2009) proved $M \mathcal{I}=s M \mathcal{I}$ for all WSVG with $n<8$, which means that the cone containing all representations of a WSVG with $n<8$ has a vertex, and showed that for $n=8$ there exist 154 WSVGs with $M \mathcal{I}=\emptyset$ and $|s M \mathcal{I}|=2$. Kurz (2012a) extended this study for $n \leq 9$. 


\subsection{Computation}

The minimum sum representations of a $\operatorname{WSVG}(N, \mathcal{W})$, i.e., the elements that belong to $s M \mathcal{I}$, are the solutions of the integer linear program:

$$
\begin{array}{ll}
\min \sum_{i=1}^{n} w_{i} ; & \\
w(S)-w(R)-1 \geq 0 & \text { for all } S \in \mathcal{W}, R \notin \mathcal{W} ; \\
w_{i} \geq 0 & \text { for all } i \in N ; \\
w_{i} \in \mathbb{Z} & \text { for all } i \in N .
\end{array}
$$

Again, $w(S)$ and $w(R)$ denote the sum of weights of voters belonging to the coalitions $S$ and $R$.

In a game with $n$ voters, there will be $2^{n}$ coalitions. The number of constraints in Problem (1) is given by:

$$
|\mathcal{W}| \cdot\left(2^{n}-|\mathcal{W}|\right)+n
$$

Consequently, the maximum number of constraints equals $2^{2 n-2}+n$. This occurs when exactly one half of all coalitions are winning. The number of effective constraints is likely to be lower, as some of them may be redundant. Most linear programming solvers can remove redundant constraints without changing the feasible region of the problem. One way of identifying redundant constraints in a linear system is discussed in Telgen (1983).

Appendix A illustrates the constraints for the game [51;49,49,2]. Table A.1 lists all coalitions of the game. Since exactly one half of the coalitions are winning, the corresponding linear integer program will have the maximum number of constraints attainable in a game with three voters. Table A.2 lists the constraints, marking the effective constraints with an asterisk. Discarding ineffective constraints, the vector $(1,1,1)$ can be discerned as the unique solution to the program. The corresponding power vector is $(1 / 3,1 / 3,1 / 3)$.

The above integer linear program involves a large number of constraints. There exist more compact formulations of this program based on equivalent classes of subsets of winning coalitions and losing coalitions, called shift-minimal winning coalitions and shift-maximal losing coalitions. We refrain from discussing these special types of coalitions to avoid the addition of unnecessary detail in Section 2. We refer the interested reader to Carreras and Freixas (1996) for definitions of shift-minimal winning coalitions and shift-maximal losing coalitions. For an application of the more compact integer linear program, see Freixas and Molinero (2010b).

Proposition 4.1-(ii) furnishes the existence of a solution to Problem (1). Yet the solution may not be unique. If it is unique, it is the least element in the partial order of component-wise comparison of the feasible solutions. Finding all solutions to an integer linear program may be difficult in practice. Jung-Fa, Ming-Hua and Yi-Chung (2008) discuss finding multiple solutions to general integer linear programs. Achterberg, Heinz and Koch (2008) propose a method for finding all solutions to an integer linear program based on a search tree.

An example of a game with several minimum sum representations with the smallest $n$ and quota $q$ is the WSVG with the two minimum sum representations:

$$
[12 ; 7,6,6,4,4,4,3,2] \text { and }[12 ; 7,6,6,4,4,4,2,3] \text {. }
$$

In this example, $1 \succ_{D} 2 \approx_{D} 3 \succ_{D} 4 \approx_{D} 5 \approx_{D} 6 \succ_{D} 7 \approx_{D} 8$. 


\section{The MSR as an Index of Voting Power}

If the MSR of a game is unique, then the MSR index is the vector of voting shares in the MSR. If the MSR is not unique, the index is given by the coordinate-wise average of the voting share in all MSRs of a game. The existence of a solution to program (1) guarantees that the MSR index of power is well-defined for WSVGs.

If $s M \mathcal{I}=M \mathcal{I}$, the MSR index is proportional to the weights of the representation lying on the vertex of the cone of integer representations. If $s M \mathcal{I} \neq M \mathcal{I}$, the MSR index is proportional to the average of the weights of the minimum sum integer representations. For instance, the MSR index for the above WSVG reads:

$$
\left(\frac{7}{36}, \frac{6}{36}, \frac{6}{36}, \frac{4}{36}, \frac{4}{36}, \frac{4}{36}, \frac{2.5}{36}, \frac{2.5}{36}\right) .
$$

Proposition 5.1 The MSR index for WSVGs satisfies the following properties: null voter, efficiency, invariance under isomorphisms and strong monotonicity.

\section{ProOF.}

(i). Null voter: From Proposition 4.1-(v) it follows that $w_{i}=0$ for every representation with minimal sum. Its average is thus zero, and $K_{i}(\mathcal{W})=0$.

(ii). Efficiency: $K$ is the average of efficient allocations, so it is efficient, too.

(iii). Invariance under isomorphisms: As the voting game is complete and weighted, any pair of players is comparable using the desirability relation. Isomorphisms preserve equi-desirable classes. An isomorphism of a complete game is an element of the group $\mathcal{S}\left(N_{1}\right) \times \cdots \times \mathcal{S}\left(N_{t}\right)$, where $N_{1}, \ldots, N_{t}$ are the equi-desirable classes of the game and $\mathcal{S}\left(N_{i}\right)$ is the symmetric group of the elements in $N_{i}$ (e.g., Carreras and Freixas 2008). Thus, for an isomorphism $\pi$ in a complete game, $\pi(i)=j$ implies $i \approx_{D} j$. Then, assuming $i \approx_{D} j$ and $w_{i}>w_{j}$ for a representation in $s M \mathcal{I}$, there exists another equivalent representation in $s M \mathcal{I}$, with $w_{i}^{\prime}=w_{j}, w_{j}^{\prime}=w_{i}$ and the remaining weights unchanged. Consequently, $K_{i}(\mathcal{W})=K_{j}(\mathcal{W})$.

(iv). Strong monotonicity: If $i \succ_{D} j$ in $\mathcal{W}$, then $w_{i}>w_{j}$ for all representation in $s M \mathcal{I}$. Thus, $K_{i}(\mathcal{W})>K_{j}(\mathcal{W})$

Proposition 5.1 establishes the coherence of the MSR index as a measure of power, as well as its ordinal equivalence to the Banzhaf, Shapley-Shubik and Johnston indices. The ordinal equivalence because, as it was shown in Freixas et al. (2012), the three indices satisfy null voter, invariance under isomorphisms and strong monotonicity, as the MSR index does. The ordinal equivalence allows us to extend some results on rankings for the three existing indices to the MSR index. One such result concerns the number of achievable hierarchies among the players.

Every CSVG, and thus every WSVG, has associated with it a hierarchy of classes of players for whom the equivalence relationship $\approx_{D}$ holds, where the classes are strictly ordered by the 
relationship $\succsim_{D}$. The number of achievable hierarchies in a WSVG with $n$ voters is given by: ${ }^{2}$

$$
\begin{aligned}
& 2^{n-1} \text { for } n=2 \\
& 2^{n-1}-1 \text { for } n=3 \\
& 2^{n-1}-2 \text { for } n \geq 4
\end{aligned}
$$

These numbers hold for the MSR index, as can be seen for $n \leq 4$ from Table B.1 of Appendix B. The table lists all possible raw power vectors for $n \leq 4$, and all WSVGs with these power vectors up to an isomorphism. The adjective 'raw' refers to an index prior to a normalization by the sum of voting weights. A comparison to raw Banzhaf and Shapley-Shubik indices shows that in games with $n \leq 4$ players the MSR index achieves fewer power distributions than the Shapley-Shubik index, which in turn achieves fewer power distributions than the Banzhaf index.

The enumeration of weighted voting games plays an important role in solving the inverse problem of finding a weighted voting game with a given distribution of power. Leech (2003) and Aziz, Paterson and Leech (2007) proposed two fixed-point iteration methods for obtaining the inverse solution for the Banzhaf index. For a general method based on linear programs, see Kurz (2012b). ${ }^{3}$

\subsection{The inverse MSR problem}

The inverse problem of a power index is the problem of designing a weighted voting game with a given distribution of power. The practical significance of this lies in institutional design. In a weighted voting game, the design is given by the voting weights and the voting rule, or the number of affirmative votes required to pass a decision. In institutions, in which the number of votes depends on the capital contributions of the voters to a purse of a given size, a voter wants to know which distributions of voting weights confer the desired power or that voter's expected share of the purse. A prime example of such an institution is the corporation.

The formulation of the inverse problem for the MSR index is considerably simpler than the formulation for the Banzhaf and Shapley-Shubik indices, but solving the inverse problem for the MSR index still requires checking all possible voting rules (quotas), and verifying that the power vector fulfills the desirability relation once a quota is fixed. The fact that power is proportional to the weights in the MSR greatly simplifies the search for a solution to the inverse problem.

Consider a variant of the inverse problem, in which we are asked to guess a game with integer weights, after being given the power vector and quota of that game. The given power vector and quota are compatible, so that we can be sure a solution to the inverse problem exists. Since there will be an infinite number of solutions, we will seek the MSR of the original game, or - if not unique - the average of the MSRs of the original game, rather than the game itself.

Let $\left(p_{1}, \ldots, p_{n}\right)$ be the power vector and $q$ the relative quota, where $p_{i} \geq 0$ for all $i=$ $1, \ldots, n, \sum_{i=1}^{n} p_{i}=1$, and $q \in(0.5,1]$ as the relative quota. Consider the weighted voting game $\left[q ; p_{1}, \ldots, p_{n}\right]$. The voting weights in this game are not integers but rationals confined to a simplex. Plugging $\left[q ; p_{1}, \ldots, p_{n}\right]$ in Problem (1) will produce an MSR of the original WSVG as the solution, because the game $\left[q ; p_{1}, \ldots, p_{n}\right]$ is equivalent to the original game and to the MSR of the original game. If the game has several MSRs, plugging $\left[q ; p_{1}, \ldots, p_{n}\right]$ in Problem (1) will yield the average of all MSRs, which is also equivalent to the original game. Indeed, for

\footnotetext{
${ }^{2}$ For a proof, see Friedman, McGrath and Parker (2006). For other papers on hierarchies in SVGs, see Carreras and Freixas (1996), Bean, Friedman and Parker (2008) and Freixas and Pons (2010).

${ }^{3}$ See, also, de Keijzer, Klos and Zhang (2010) and Alon and Edelman (2010).
} 
a given input $\left[q ; p_{1}, \ldots, p_{n}\right]$, there exists a unique integer $\lambda>0$, such that $\left(\lambda p_{1}, \lambda p_{2}, \ldots, \lambda p_{n}\right)$ is the average of all MSRs of the solution. Since rescaling the weights and quota leaves the set of winning coalitions unchanged, $\left[\lambda q ; \lambda p_{1}, \lambda p_{2}, \ldots, \lambda p_{n}\right]$ is the solution. As an example, consider the power vector

$$
\left(\frac{14}{72}, \frac{12}{72}, \frac{12}{72}, \frac{8}{72}, \frac{8}{72}, \frac{8}{72}, \frac{5}{72}, \frac{5}{72}\right)
$$

After multiplying the above power vector by $\lambda=36$, we obtain $(7,6,6,4,4,4,2.5,2.5)$, which is the average of the weights of the two MSRs for the game discussed in Section 4.

The difference between the above problem and the actual inverse problem is that in the latter we are given the power vector but not the quota. If we know that $\left(p_{1}, \ldots, p_{n}\right)$ is a power vector of a WSVG, then we also know that there are at most $2^{n}-1$ candidates to represent a game with this power vector. Candidates for the quota are:

$$
\sum_{i \in S} p_{i} \text { for all } S \subseteq N, S \neq \emptyset .
$$

If the WSVG obtained by choosing a given quota preserves the desirability relation, then it is a solution to the inverse problem. Otherwise the quota should be discarded in favor of a different one.

The next section provides examples of power distributions obtained using the MSR index, and compares them to the widely-applied Banzhaf and Shapley-Shubik indices.

\section{$6 \quad$ Numerical Examples}

Example 1 (IMF). The first example applies the MSR index to the country voting in two constituencies of the International Monetary Fund (IMF). The two constituencies are currently led by Belgium and Mexico (as of the first half of 2012, subject to a biannual rotation scheme). ${ }^{4}$ We assume simple majority as the voting rule.

Table 1: Power distribution in the IMF Belgium-led constituency.

\begin{tabular}{lcccccc}
\hline \hline Country & Weight & MSR & MSR Index & Imp. Weight & Banzhaf & Shapley-Shubik \\
\hline Austria & 21880 & 13 & 0.149 & 18713 & 0.101 & 0.132 \\
Belarus & 4605 & 3 & 0.034 & 4318 & 0.027 & 0.026 \\
Belgium & 46793 & 31 & 0.356 & 44623 & 0.530 & 0.491 \\
Czech Republic & 10763 & 8 & 0.092 & 11515 & 0.067 & 0.060 \\
Hungary & 11125 & 9 & 0.103 & 12955 & 0.081 & 0.080 \\
Kosovo & 1331 & 2 & 0.023 & 2879 & 0.017 & 0.019 \\
Luxembourg & 4928 & 4 & 0.046 & 5758 & 0.032 & 0.034 \\
Slovak Republic & 5016 & 4 & 0.046 & 5758 & 0.032 & 0.034 \\
Slovenia & 3491 & 2 & 0.023 & 2879 & 0.017 & 0.019 \\
Turkey & 15299 & 11 & 0.126 & 15834 & 0.096 & 0.104 \\
\hline Gini Coef. & & 0.457 & & 0.573 & 0.560 \\
\hline \hline
\end{tabular}

Simple majority quota: 62616 votes.

\footnotetext{
${ }^{4}$ For an comprehensive analysis of country voting in the International Monetary Fund, the World Bank and its three regional equivalents in America, Africa and Asia using existing power indices, see Leech and Leech (2005) and Strand (1999, 2001, 2003).
} 
Tables 1 and 2 summarize the results. Compared to the Banzhaf and Shapley-Shubik indices, the MSR index assigns less power to the largest player (Belgium) and more power to the smallest players (Kosovo and Slovenia). The Gini coefficient confirms that voting power according to the MSR index is less concentrated. In the sense of the sum of absolute deviations, the MSR power vector is closer to the Shapley-Shubik index (0.27) than to the Banzhaf index (0.35).

The number of votes a country commands is determined by its contribution to the authorized ordinary capital of the IMF. Using the voting powers obtained with the MSR index as a basis for determining fair capital contributions leads to the implicit voting weights shown in the fifth column of Table 1. It turns out that Austria, Belarus, Belgium and Slovenia contribute too much relative to the voting power they receive, whereas all other countries contribute too little.

The power distribution the Mexican-led constituency is very uneven. The three larger members share the power equally, whereas the smaller members are powerless. The power distribution according to the MSR index is identical to those according to the other two indices.

Table 2: Power distribution in the IMF Mexico-led constituency.

\begin{tabular}{lccccc}
\hline \hline Country & Weight & MSR & MSR Index & Banzhaf & Shapley-Shubik \\
\hline Costa Rica & 2382 & 0 & 0 & 0 & 0 \\
El Salvador & 2454 & 0 & 0 & 0 & 0 \\
Guatemala & 2843 & 0 & 0 & 0 & 0 \\
Honduras & 2036 & 0 & 0 & 0 & 0 \\
Mexico & 36998 & 1 & 0.333 & 0.333 & 0.333 \\
Nicaragua & 2041 & 0 & 0 & 0 & 0 \\
Spain & 40975 & 1 & 0.333 & 0.333 & 0.333 \\
Venezuela & 27332 & 1 & 0.333 & 0.333 & 0.333 \\
\hline \hline
\end{tabular}

Simple majority quota: 58531 votes.

Example 2 (Bundestag). Our second example considers the distribution of parliamentary seats in the German Bundestag after the general election of 2009. Popular methods for allocating a fixed number of seats in way that best represents the shares of the popular vote obtained by the parties include the D'Hondt formula, the Droop quota and the Largest Remainder Method. The Bundestag currently uses the Sainte-Laguë method.

Table 3: German Bundestag election, 2009.

\begin{tabular}{lccccc}
\hline \hline Party & Popular Vote & Seats & MSR & MSR Index & Imp. Seats \\
\hline CDU/CSU & $14,655,004$ & 239 & 3 & 0.333 & 207 \\
SPD & $9,988,843$ & 146 & 2 & 0.222 & 138 \\
FDP & $6,313,023$ & 93 & 2 & 0.222 & 138 \\
The Left & $5,153,884$ & 76 & 1 & 0.111 & 69 \\
Green & $4,641,197$ & 68 & 1 & 0.111 & 69 \\
\hline
\end{tabular}

An alternative would be to allocate parliamentary seats according to the voting power implied in the popular vote in a way that minimizes the total number of seats in the parliament and, hence, also the monetary cost of indirect representation. This can be accomplished using the 
MSR index. Table 3 summarizes computations for the Bundestag after the general election of 2009. The power distribution implied in the popular vote can be captured using just nine seats, three of which go to the CDU/CSU as the party with the most popular votes. Note that minimizing the cost of representation may also minimize its quality. There are good reasons for having sizable parliaments, such as the number and complexity of issues, regional representation, etc. So, if one wishes to adhere to a 622 seat Bundestag, the seat distribution implied in voting power bestowed by the popular vote will be as in the rightmost column of Table 3 .

\section{Further Properties of the MSR index}

One of the difficult questions in the empirical work is which index to use. The common criteria for choosing an index include the existence of more or less compelling game-theoretical foundations (Shapley-Shubik, Deegan-Packel), consistency with certain stochastic models of voting (ShapleyShubik, absolute Banzhaf), or immunity to certain voting paradoxes.

We already mentioned that the Deegan-Packel and Holler indices are not coherent measures of power because they violate monotonicity. The absolute Banzhaf measure violates efficiency, which makes it a dubious measure of P-power. This leaves the Banzhaf, Johnston and ShapleyShubik indices as reasonable measures of P-power. Felsenthal and Machover (1998) identify three voting paradoxes that any reasonable measure of power should not be liable to. These are the bloc, donation and bicameral meet paradoxes. The problem is that none of the existing measures of power have all the required immunities (Table 4), leaving open the question of which index to use.

Table 4: Properties of Power Indexes.

\begin{tabular}{lcccccccc}
\hline \hline Index & Null & Eff. & Invar. & Str.Mon. & Bloc & Neut. & Don. & Bic.Meet \\
\hline Shapley and Shubik (1954) & $\checkmark$ & $\checkmark$ & $\checkmark$ & $\checkmark$ & $\checkmark$ & & $\checkmark$ & \\
Banzhaf (1965) & $\checkmark$ & $\checkmark$ & $\checkmark$ & $\checkmark$ & & & & $\checkmark$ \\
Johnston (1978) & $\checkmark$ & $\checkmark$ & $\checkmark$ & $\checkmark$ & & & & \\
Deegan and Packel (1978) & $\checkmark$ & $\checkmark$ & $\checkmark$ & & & & & \\
Holler (1982) & $\checkmark$ & $\checkmark$ & $\checkmark$ & & & & & \\
MSR & $\checkmark$ & $\checkmark$ & $\checkmark$ & $\checkmark$ & & $\checkmark$ & & $\checkmark$ \\
\hline \hline
\end{tabular}

Respecting the bloc postulate means that if two voters or more voters form a bloc by adding their votes, the power of the bloc should not be lower than the power of either voter alone. A weaker version of the bloc postulate concerns symmetric games. In a symmetric weighted voting game, each player commands an equal number of votes. The power of a voting bloc according to the MSR index equals the sum of individual powers of its members, so that satisfying the bloc postulate does not carry strategic implications. We call this property Neutrality. None of the popular existing indices satisfy this property (Table 5).

Respecting donation means that if one voter gives some of her votes to another, the power of the donor should not increase as a result. Felsenthal and Machover (1998) provide examples in which the Banzhaf and Johnston indices display both bloc and donation paradoxes. Freixas and Molinero (2010a) study the frequency of the occurrence of the donation paradox in weighted games with a small number of players, providing examples for the Banzhaf and Johnston indices.

The MSR index is also liable to bloc and donation paradoxes. The Shapley-Shubik index is immune to both bloc and donation paradoxes, but it does not satisfy the bicameral meet 
satisfied by the Banzhaf and MSR indexes.

An index of power respects bicameral meet if the ratio of powers of any two voters belonging to the same assembly prior to a merge with a different assembly is preserved in the joint assembly. This property is useful when measuring voting power of shareholders, because the relative powers of shareholders comprising a minority voting assembly (represented by their joint holdings) carry over to the grand voting assembly (represented by the total worth of the company).

To prove that the MSR index respects bicameral meet we first need to establish the conditions under which a bicameral meet of two complete games is complete, and a bicameral meet of two weighted games is weighted. This is accomplished in the next section.

Table 5: Bloc and Donation Paradoxes in games $[22 ; 10,9,7,6,4,1]$ and $[22 ; 10,9,7,6,5,0]$.

\begin{tabular}{lcccccc}
\hline \hline Voter & Weight & MSR Index & Shapley-Shubik & Weight & MSR Index & Shapley-Shubik \\
\hline 1 & 10 & 0.280 & 0.300 & 10 & 0.308 & 0.317 \\
2 & 9 & 0.240 & 0.250 & 9 & 0.231 & 0.233 \\
3 & 7 & 0.200 & 0.217 & 7 & 0.231 & 0.233 \\
4 & 6 & 0.160 & 0.167 & 6 & 0.154 & 0.150 \\
5 & 4 & 0.080 & 0.050 & 5 & 0.077 & 0.067 \\
6 & 1 & 0.040 & 0.017 & 0 & 0 & 0 \\
\hline \hline
\end{tabular}

Bloc Paradox: In the game [22;10,9,7,6,4,1], voter 5 gains power by forming a bloc with voter 6 according to the Shapley-Shubik index, but loses power according to the MSR index.

Donation Paradox: In the game $[22 ; 10,9,7,6,5,0]$, voter 5 loses power by donating one vote to voter 6 according to the Shapley-Shubik index, but gains power according to the MSR index.

\subsection{Two characterizations for bicameral meet games}

The bicameral meet of two SVGs $\left(N_{1}, \mathcal{W}_{1}\right)$ and $\left(N_{2}, \mathcal{W}_{2}\right)$ with disjoint assemblies is an SVG such that $N=N_{1} \cup N_{2}$ and $\mathcal{W}=\left\{S \subseteq N: S=S_{1} \cup S_{2}, S_{1} \in \mathcal{W}_{1}, S_{2} \in \mathcal{W}_{2}\right\}$. The bicameral meet postulate requires that if $i$ and $j$ are non-null voters in an SVG $\left(N_{1}, \mathcal{W}_{1}\right)$, then the ratio of power of voter $i$ to the power of voter $j$ in the joint assembly $N_{1} \cup N_{2}$ should be equal to the ratio of their powers in the original game $\left(N_{1}, \mathcal{W}_{1}\right)$ :

$$
\frac{K_{i}\left(\mathcal{W}_{1}\right)}{K_{j}\left(\mathcal{W}_{1}\right)}=\frac{K_{i}(\mathcal{W})}{K_{j}(\mathcal{W})}
$$

A special case of the bicameral meet postulate is the added blocker postulate, which says that adding a vetoer to a WSVG should not change the ratio of powers of any two incumbent voters.

Felsenthal, Machover and Zwicker (1998) proved that the Banzhaf index satisfies bicameral meet, while the Deegan-Packel, Johnston and Shapley-Shubik indices do not. A violation for the Holler index can be obtained using their example for the Deegan-Packel index.

The bicameral meet of two complete games may not be complete, and the bicameral meet of two weighted games may not be weighted. The next two theorems provide characterizations for complete and weighted bicameral meets. 
Theorem 7.1 Let $(N, \mathcal{W})$ be the bicameral meet of $\left(N_{i}, \mathcal{W}_{i}\right)$ for $i=1,2$. Then, $(N, \mathcal{W})$ is a complete game if and only if $\left(N_{i}, \mathcal{W}_{i}\right)$ for $i=1,2$ are complete games, and at least one of them is a unanimity of a coalition game.

Proof.

$(\Rightarrow)$ Assume that $(N, \mathcal{W})$ is a complete game and that none of the two games $\left(N, \mathcal{W}_{i}\right)$ is a unanimity of a coalition game. According to the definition of a unanimity of a coalition game, there is a voter $i \in N_{1}$, who is neither vetoer nor null in $\left(N_{1}, \mathcal{W}_{1}\right)$, and a voter $j \in N_{2}$, who is neither vetoer nor null in $\left(N_{2}, \mathcal{W}_{2}\right)$. This implies the existence of coalitions

$$
\begin{array}{lll}
S_{1}, T_{1} & \text { with } & i \in S_{1} \in \mathcal{W}_{1}^{m}, i \notin T_{1} \in \mathcal{W}_{1}^{m} \\
S_{2}, T_{2} & \text { with } & j \in S_{2} \in \mathcal{W}_{2}^{m}, j \notin T_{2} \in \mathcal{W}_{2}^{m},
\end{array}
$$

such that $S_{1} \cup T_{2}$ and $S_{2} \cup T_{1}$ are both minimally winning in $(N, \mathcal{W})$, but $\left(S_{1} \backslash\{i\}\right) \cup\left(T_{2} \cup\{j\}\right)$ and $\left(S_{2} \backslash\{j\}\right) \cup\left(T_{1} \cup\{i\}\right)$ are both losing in $(N, \mathcal{W})$. This shows that $(N, \mathcal{W})$ is not swap robust, and thus not complete. However, this contradicts the initial assumption.

Consequently, one of the two games $\left(N, \mathcal{W}_{i}\right)$ for $i=1,2$ is a unanimity game of a coalition. Let $\left(N_{2}, \mathcal{W}_{2}\right)$ be such game. Then, $\mathcal{W}_{2}^{m}=\{T\}$. This game is complete because players in $T$ are vetoers in $\left(N_{2}, \mathcal{W}_{2}\right)$, and players in $N_{2} \backslash T$ are nulls in $\left(N_{2}, \mathcal{W}_{2}\right)$. The completeness of $\left(N_{2}, \mathcal{W}_{2}\right)$ follows from null players being dominated according to Isbell's desirability relation by veto players.

It remains to show that $\left(N_{1}, \mathcal{W}_{1}\right)$ is complete. Assume that $i \succsim_{D} j$ in $(N, \mathcal{W})$, with $i, j \in N_{1}$. We want to prove that $i \succsim_{D} j$ in $\left(N_{1}, \mathcal{W}_{1}\right)$. Suppose, to the contrary, that there exists a coalition $S_{1} \subseteq N_{1} \backslash\{i, j\}$, such that $S_{1} \cup\{j\} \in \mathcal{W}_{1}$ but $S_{1} \cup\{i\} \notin \mathcal{W}_{1}$. Then, $S_{1} \cup\{j\} \in \mathcal{W}_{1}$ implies $\left(S_{1} \cup S_{2}\right) \cup\{j\} \in \mathcal{W}$ for all $S_{2} \in \mathcal{W}_{2}$, which implies $\left(S_{1} \cup S_{2}\right) \cup\{i\} \in \mathcal{W}$ since $i \succsim_{D} j$ in $(N, \mathcal{W})$. Hence $S_{1} \cup\{i\} \in \mathcal{W}_{1}$ is a contradiction.

$(\Leftarrow)$ Assume, without any loss of generality, that $\left(N_{2}, \mathcal{W}_{2}\right)$ is the unanimity game of coalition $T$ for some $\emptyset \subset T \subseteq N_{2}$. Players in $T$ have veto in $\left(N_{2}, \mathcal{W}_{2}\right)$, while players in $N_{2} \backslash T$ are nulls in $\left(N_{2}, \mathcal{W}_{2}\right)$. Since veto players dominate null players, $\left(N_{2}, \mathcal{W}_{2}\right)$ must be a complete game.

Assume, moreover, that $\left(N_{1}, \mathcal{W}_{1}\right)$ is a complete game with the following total (weak) order: $1 \succsim_{D} 2 \succsim_{D} \cdots \succsim_{D} n_{1}$. Let $N=\left\{1, \ldots, n_{1}, n_{1}+1, \ldots, n_{1}+t, n_{1}+(t+1), \ldots, n_{1}+n_{2}\right\}$ with $n_{1}+n_{2}=n$, so that $j \in T$ if $j=n_{1}+i$ for some $1 \leq i \leq t$. Since $N=N_{1} \cup N_{2}$ and

$$
\mathcal{W}=\left\{S_{1} \cup S_{2}: S_{1} \in \mathcal{W}_{1}, S_{2} \in \mathcal{W}_{2}\right\}
$$

players in $T$ have veto in $(N, \mathcal{W})$. Players in $T$ therefore dominate all players in $N$. On the contrary, players in $N_{2} \backslash T$ are null in $(N, \mathcal{W})$ and are therefore dominated by all players in $N$. In game $(N, \mathcal{W})$ we thus have

$$
n_{1}+1 \approx_{D} \cdots \approx_{D} n_{1}+t \succsim_{D} 1 \succsim_{D} 2 \succsim_{D} \cdots \succsim_{D} n_{1} \succsim_{D} n_{1}+(t+1) \approx_{D} \cdots \approx_{D} n_{1}+n_{2} .
$$

Consequently, $(N, \mathcal{W})$ is a complete game, with at least $t$ players being the most desirable and at least $n_{2}-t$ being the least desirable in $(N, \mathcal{W})$.

Corollary 7.2 If $(N, \mathcal{W})$ is a CSVG which is the bicameral meet of $\left(N_{1}, \mathcal{W}_{1}\right)$ and $\left(N_{2}, \mathcal{W}_{2}\right)$, then either $\left|\mathcal{W}_{1}^{m}\right|=1$ or $\left|\mathcal{W}_{2}^{m}\right|=1$, and, therefore, $\left|\mathcal{W}^{m}\right|=\left|\mathcal{W}_{1}^{m}\right| \cdot\left|\mathcal{W}_{2}^{m}\right|$.

Note that Corollary 7.2 also applies to weighted games. Another direct consequence of Theorem 7.1 is that if $(N, \mathcal{W})$ is a complete game without veto players, then it cannot be the bicameral meet of two SVG. 
Theorem 7.3 Let $(N, \mathcal{W})$ be the bicameral meet of $\left(N_{i}, \mathcal{W}_{i}\right) i=1,2$. Then, $(N, \mathcal{W})$ is a weighted game, if and only if $\left(N_{i}, \mathcal{W}_{i}\right)$ for $i=1,2$ are weighted games, of which one is a unanimity of a coalition game.

Proof. $(\Rightarrow)$ Assume that $(N, \mathcal{W})$ is a weighted game. By Theorem 7.1 , we only need to prove that $\left(N_{1}, \mathcal{W}_{1}\right)$ is a weighted game, as $[t ; \underbrace{1, \ldots, 1}_{t}, \underbrace{0, \ldots, 0}_{n_{2}-t}]$ is a weighted representation for the unanimity of a coalition game $T \subseteq N_{2},\left(N_{2}, \mathcal{W}_{2}\right)$.

Let $[q ; w] \equiv\left[q ; w_{1}, \ldots, w_{n_{1}}, w_{n_{1}+1}, \ldots, w_{n_{1}+n_{2}}\right]$ be a weighted representation for $(N, \mathcal{W})$. We claim that

$$
\left[q^{\prime} ; w^{\prime}\right] \equiv\left[q-\sum_{i \in T} w_{i} ; w_{1}, \ldots, w_{n_{1}}\right]
$$

is a representation for $\left(N_{1}, \mathcal{W}_{1}\right)$. We need to prove that

$$
S_{1} \in \mathcal{W}_{1} \Longleftrightarrow w^{\prime}\left(S_{1}\right) \geq q^{\prime}
$$

This follows from the following two steps:

- If $S_{1} \in \mathcal{W}_{1}$, then $S_{1} \cup T \in \mathcal{W}$. This implies $w\left(S_{1} \cup T\right) \geq q$, and, consequently, $w^{\prime}\left(S_{1}\right) \geq$ $q-\sum_{i \in T} w_{i}=q^{\prime}$

- If $S_{1} \notin \mathcal{W}_{1}$, then $S_{1} \cup N_{2} \notin \mathcal{W}$. This implies $w\left(S_{1} \cup N_{2}\right)<q$, and, consequently, $w^{\prime}\left(S_{1}\right) \leq$ $w\left(S_{1} \cup N_{2}\right)-\sum_{i \in T} w_{i}<q-\sum_{i \in T} w_{i}=q^{\prime}$.

$(\Leftarrow)$ Let $[t ; \underbrace{1, \ldots, 1}_{t}, \underbrace{0, \ldots, 0}_{n_{2}-t}]$ be a weighted representation for $\left(N_{2}, \mathcal{W}_{2}\right)$ and $\left[q ; w_{1}, \ldots, w_{n_{1}}\right]$ be a weighted representation for $\left(N_{1}, \mathcal{W}_{1}\right)$. Then we claim that

$$
\left[q^{\prime \prime} ; w^{\prime \prime}\right] \equiv[q+t v ; w_{1}, \ldots, w_{n_{1}}, \underbrace{v, \ldots, v}_{t}, \underbrace{0, \ldots, 0}_{n_{2}-t}]
$$

where $v=w\left(N_{1}\right)-q+1$ is a weighted representation for $(N, \mathcal{W})$. Indeed, we need to prove that

$$
S \in \mathcal{W} \quad \Longleftrightarrow w^{\prime \prime}(S) \geq q^{\prime \prime} .
$$

This follows from the following two steps:

- If $S \in \mathcal{W}$, then $S \cap N_{1} \in \mathcal{W}_{1}$ and $S \cap N_{2} \in \mathcal{W}_{2}$, thus $w^{\prime \prime}\left(S \cap N_{1}\right) \geq q$ and $w^{\prime \prime}\left(S \cap N_{2}\right) \geq t v$ since $T \subseteq S$, which implies $w^{\prime \prime}(S)=w^{\prime \prime}\left(S \cap N_{1}\right)+w^{\prime \prime}\left(S \cap N_{2}\right) \geq q+t v=q^{\prime \prime}$;

- If $S \notin \mathcal{W}$, then either $S \cap N_{1} \notin \mathcal{W}_{1}$ or $S \cap N_{2} \notin \mathcal{W}_{2}$. Assume first $S \cap N_{1} \notin \mathcal{W}_{1}$, then $w^{\prime \prime}\left(S \cap N_{1}\right)<q$, which implies $w^{\prime \prime}(S)=w^{\prime \prime}\left(S \cap N_{1}\right)+w^{\prime \prime}\left(S \cap N_{2}\right)<q+t v=q^{\prime \prime}$. Assume now $S \cap N_{2} \notin \mathcal{W}_{2}$, then $w^{\prime \prime}\left(S \cap N_{2}\right) \leq(t-1) v$, which implies $w^{\prime \prime}(S)=w^{\prime \prime}\left(S \cap N_{1}\right)+w^{\prime \prime}\left(S \cap N_{2}\right) \leq$ $w\left(N_{1}\right)+(t-1) v=q+(t-1) v+\left(w\left(N_{1}\right)-q\right)=q+(t-1) v+(v-1)=q+t v-1<q+t v=q^{\prime \prime}$.

Theorem 7.3 implies that if $(N, \mathcal{W})$ is a weighted voting game without veto players, then it cannot be the bicameral meet of two simple voting games. 


\subsection{The MSR index respects bicameral meet}

To prove that the MSR index respects bicameral meet, we need the following lemma:

Lemma 7.4 Let $(N, \mathcal{W})$ be the bicameral meet of the two weighted games $\left(N_{i}, \mathcal{W}_{i}\right)$ for $i=1,2$ where $\left(N_{2}, \mathcal{W}_{2}\right)$ is a unanimity of a coalition game. The elements of $s M \mathcal{I}\left(\mathcal{W}_{1}\right)$ and $s M \mathcal{I}(\mathcal{W})$ are in bijective correspondence. Moreover, this one-to-one correspondence preserves weights.

Proof. Assume, without any loss of generality, that $\left(N_{2}, \mathcal{W}_{2}\right)$ has trivial voters only. Then, $\left(N_{2}, \mathcal{W}_{2}\right)$ admits the weighted representation $[t ; \underbrace{1, \ldots, 1}_{t}, \underbrace{0, \ldots, 0}_{n_{2}-t}]$ for some $1 \leq t \leq n_{2}$, which is the unique element in $s M \mathcal{I}\left(\mathcal{W}_{2}\right)$. Let $\left[q ; w_{1}, \ldots, w_{n_{1}}\right]$ be an element of $s M \mathcal{I}\left(\mathcal{W}_{1}\right)$. Then we claim that

$$
[q+t v ; w_{1}, \ldots, w_{n_{1}}, \underbrace{v, \ldots, v}_{t}, \underbrace{0, \ldots, 0}_{n_{2}-t}]
$$

where $v=w\left(N_{1}\right)-q+1$, is an element of $s M \mathcal{I}(\mathcal{W})$.

Assume, on the contrary, that the above representation does not belong to $s M \mathcal{I}(\mathcal{W})$. Then we could decrease at least one weight in a manner that leaves the set of minimal winning coalitions $\mathcal{W}$ unchanged. We now show that doing so produces a contradiction.

Subtracting one vote from any of the first $n_{1}$ weights yields $\left[q ; w_{1}, \ldots, w_{n_{1}}\right] \notin s M \mathcal{I}\left(\mathcal{W}_{1}\right)$, which is a contradiction.

Subtracting one vote from any of the middle $t$ weights produces a contradiction with the assumption of that voter having a veto in $\mathcal{W}_{2}$. Indeed, by Proposition 4.1-(iv) there exists $S \in \mathcal{W}_{1}^{m}$, such that $w(S)=q$. In this case, $S \cup N_{2} \notin \mathcal{W}$, as $w\left(S \cup N_{2}\right)<q+t v$.

If the quota $q+t v$ is decreased by one, then, according to Proposition 4.1-(v), the remaining weights $v$ must be decreased by one vote to preserve the minimum sum property. This implies that the quota must be lowered to $q+t v-t(t \geq 1)$. Consequently, by Proposition 4.1-(iv), the quota for the initial weighted representation of game $\left(N, \mathcal{W}_{1}\right)$ could be lowered to $q-t$. This contradicts the existence of a losing coalition with weight $q-1$ implied in Proposition 4.1-(iv).

The proof that the MSR index respects bicameral meet follows readily from the above lemma. Since the weights in the bijective map established in Lemma 7.4 for non-null voters do not change, we conclude that if $i, j \in N_{1}$, then

$$
\frac{K_{i}\left(\mathcal{W}_{1}\right)}{K_{j}\left(\mathcal{W}_{1}\right)}=\frac{K_{i}(\mathcal{W})}{K_{j}(\mathcal{W})}
$$

The examples given in Felsenthal et al. (1998), showing that the Deegan-Packel, Johnston and Shapley-Shubik indices do not satisfy the bicameral meet property, are examples of bicameral meets in WSVGs. Thus, although these three indices are defined for more general SVGs, they do not respect the bicameral meet property in WSVGs, whereas the MSR index does.

\subsection{The MSR index is neutral for blocs in symmetric games}

Consider a simple weighted voting game with $n$ voters, each of whom has exactly one vote. The decision rule is defined by a threshold number of votes, $q$. In this simple setting, any index that respects the invariance and efficiency properties will ascribe $1 / n$ to each voter. But now suppose 
that $b$ voters form a voting bloc. What is the relationship between the power of the bloc and the sum of the individual voting powers of its members?

Let us assume that the bloc does not command the required majority of votes, i.e., $1<$ $b<q \leq n-b$. Under this assumption, Straffin (1980) proves that the Shapley-Shubik index of the bloc equals $b /(n-b+1)$, which is greater than the sum of powers of the individual voters $b / n$. It is clear from the integer linear program (1) that the weighted representation of the game $[q ; b, \underbrace{1, \ldots, 1}_{n-b}]$ is minimum in integers, so that $(b, \underbrace{1, \ldots, 1}_{n-b})$ is the distribution of power according to the MSR index for the game obtained after the formation of the bloc. Thus, the MSR index fulfills the bloc postulate in its neutral form.

None of the other indices in Table 5 has this property. The Banzhaf index of the bloc reads

$$
\frac{B(n, q, b)}{B(n, q, b)+(n-b) A(n, q, b)}
$$

where $B(n, q, b)$ and $A(n, q, b)$ denote, respectively, the number of swings for the bloc and the number of swings for a single member of the bloc in the game obtained after forming the bloc. It is easy to show that

$$
B(n, q, b)=\sum_{k=0}^{b-1}\left(\begin{array}{c}
n-b \\
q-b+k
\end{array}\right) \quad \text { and } \quad A(n, q, b)=\left(\begin{array}{c}
n-b-1 \\
q-1
\end{array}\right)+\left(\begin{array}{c}
n-b-1 \\
q-b-1
\end{array}\right)
$$

For $n<8$, the power of the bloc is greater than the sum of individual powers of its members. Yet, for example, for $n \geq 8, b=2$ and $q=n-2$, the power of the bloc is smaller than $2 / n$, the sum of powers of any two voters. This shows that the Banzhaf index neither satisfies the bloc postulate, nor the neutrality satisfied by the MSR index. We leave the computations for the other three indices to the reader.

\subsection{The MSR index for games with a priori unions}

The bloc postulate motivate comparisons of power distributions in two games: the initial game and the one obtained after the formation of a bloc. More general games with voting blocs can be obtained by partitioning the set $N$ of players into a priori unions $\mathbf{T}=\left\{T_{1}, \ldots, T_{m}\right\}$, where the coalitions (unions) $T_{k}$ are pairwise disjoint, and their union is $N$. These unions are seen as prior agreements among a group of players. The central questions in the context of games with voting blocs are: (i) what is the power of the bloc, and (ii) how to share the spoils among the bloc members. ${ }^{5}$

Given a partition $\mathbf{T}$, the quotient game $\mathcal{V}=\mathcal{W} / \mathbf{T}$ with players set $M=\{1, \ldots, m\}$, assigns to each bloc the value of the MSR index in the quotient game, i.e., $\mathcal{V} \equiv\left[q ; w\left(T_{1}\right), \ldots, w\left(T_{m}\right)\right]$ where $w(S)=\sum_{i \in S} w_{i}$. We compute the MSR index for this game and divide the value obtained for the bloc among its members proportionally to the value that these players receive according to the MSR index in the initial game.

While providing an axiomatization of this definition is beyond the scope of this paper, we note that from a computational viewpoint this definition requires solving two integer linear programs instead of one: the program associated to the original game and the program associated to the

\footnotetext{
${ }^{5}$ There exists an extensive literature on coalitional values for games with a priori unions based on the indices considered in this paper. See, Owen (1977) for a seminal contribution on games with a priori and, e.g., AlonsoMeijide and Fiestras-Janeiro (2002) and Pulido and Sánchez-Soriano (2009) for more recent work.
} 
quotient game. To give a simple example, consider the game $[3 ; 2,1,1]$. If $\mathbf{T}=(\{1,2\},\{3\})$, then player 3 becomes null, whereas players 1 and 2 share the spoils in proportion $2: 1$, yielding the power vector $(2 / 3,1 / 3,0)$. If $\mathbf{T}=(\{1\},\{2,3\})$, then all players are equally powerful in the quotient game, so that they get $1 / 2$ each. As 2 and 3 receive $1 / 4$ each for the MSR index in the initial game, the $1 / 2$ assigned in the quotient game is divided equally between them. The final distribution of power is $(1 / 2,1 / 4,1 / 4)$.

\section{Concluding Remarks}

Briefly summarizing our contribution, we have developed a new index of voting power based on the Minimum Sum Representation (MSR) of a weighted voting game. We have established that the MSR index, computed as the voter share in the sum of weights of the minimum sum representation, is a coherent measure of power. In addition, the MSR index is immune to the bicameral meet paradox.

The MSR is an interesting object in its own right. It offers a redesign of the original game, in which power becomes proportional to voting weight. The observation that power is not proportional to weight in general, or that the distribution of votes reveals little about the distribution of power, has been one of the main reasons for developing power indices in the past. Of course, the total sum of weights in a minimum sum representation would usually be different than in the original game, in which case a rescaling of the quota and weights would be necessary in order to restore the original sum of weights. However, that would not affect the distribution of power. The sum of the rescaled weights may be larger than the original sum, but the discrepancy can be kept at a minimum, if desired.

The above properties suggest the MSR representation is an optimal design for weighted voting games, in which power is proportional to weight. The drawback is that computing the MSR and the MSR index of power requires solving a large-scale integer linear program, and finding all solutions to that problem, if several exist. In this case, the index is given by a coordinate-by-coordinate average of power vectors in all MSR games. Finding all solutions to an integer linear program may require special techniques, but a solution always exists.

The inverse problem of a power index is central to designing voting institutions with a given distribution of voting power. Solving the above problem for the MSR index is as easy as computing the index, because the inverse problem is solved with the same method used to compute the index. This stands in stark contrast to the existing power indices, whose inverse problems are significantly more difficult than direct computations. The inverse problem arises in institutional design. A potentially interesting application of the MSR index is the problem of apportioning parliamentary seats after a general election in a way what minimizes the number of seats in the parliament and the resulting cost of indirect representation. Here we assume that the cost of representation is proportional to the total number of seats, which is minimized by a minimum sum representation of the game.

It is evident that in any WSVG the number of permutations in which a player is pivotal in the raw Shapley-Shubik index is greater than the number of voting outcomes in which the player is critical in the raw Banzhaf index. We conjecture that the largest possible weight a player can have in a minimum sum representation is lower than the corresponding raw Banzhaf value, and, hence, also lower than the corresponding raw Shapley-Shubik value. If true, this would imply that Banzhaf and Shapley-Shubik indices do not minimize the cost of representation.

In terms of the conventional properties of power indexes, the MSR index is quite similar 
to the Banzhaf index. However, the MSR offers two substantial advantages over any known measure of voting power, including the popular Banzhaf index and the Shapley-Shubik index. It offers way of redistributing a fixed number of total votes so that the power ascribed to a voter by the MSR becomes proportional to the share of the number of votes she commands. This proportionality of voting power and weight is attractive, both in theoretical and applied work.

Proportionality makes the MSR index an ideal measure of P-power in institutions, in which the votes are distributed to the voter based on their contribution to a fixed purse. P-power reflects the extent of a voter's control of the distribution of a fixed purse - the ultimate outcome of voting, measured by that voter's expected share in the fixed purse (Felsenthal and Machover 2004). If a voter's expected share of spoils coincides with the voters contribution to the fixed purse, an equilibrium emerges in which voters will not want to redistribute votes. This leads to a stable institutional design of vote-for-money institutions such as the corporation.

We conclude the paper with a remark on an alternative power index based on the MSR and an open problem. A different measure of power can be developed by taking the average of weight vectors for minimal sum integer weighted representations preserving types. This means that we only take into consideration weighted representations in which voters equivalent in terms of the desirability relation have the equal weights. In Freixas and Molinero (2010b) it is proved that $n=9$ is the smallest number of voters needed to find examples of WVSGs without a unique minimal sum representation preserving types. For instance, the example given in Section 5, which only has 8 voters without a unique minimal sum representation in integers, has a unique minimal sum representation in integers preserving types. However, if $n>8$ one can find examples, such as the one given in Freixas and Molinero (2010b), which admits

$$
[37 ; 14,11,7,5,5,5,3,2,2] \text { and }[37 ; 14,12,6,5,5,5,3,2,2]
$$

as minimum sum integer representations preserving types. In this example, the average of weights is

$$
\left(\frac{14}{54}, \frac{11.5}{54}, \frac{6.5}{54}, \frac{5}{54}, \frac{5}{54}, \frac{5}{54}, \frac{3}{54}, \frac{2}{54}, \frac{2}{54}\right),
$$

which also provides a suitable alternative measure with similar properties to those studied in this paper for the MSR index. Note, however, that for $n<8$ the value of this alternative index coincides with the MSR index developed in this paper.

An open problem is how to extend the MSR index from the domain of weighted voting games to the domain of simple voting games. We surmise that several alternative ways of accomplishing this might be available, but at present none of them seems simple and convincing. Perhaps some notion of dimension for simple voting games, such as those introduced in Freixas and Marciniak (2009), may offer a useful starting point.

\section{Acknowledgements}

We are greatly indebted to the three referees. Their numerous comments and suggestions helped us to write a more polished and interesting paper.

\section{References}

Achterberg, T., Heinz, S. and Koch, S.: 2008, Counting solutions of integer programs using unrestricted subtree detections, in P. Laurent and M. A. Trick (eds), Integration of AI and OR Techniques in Constraint Programming for Combinatorial Optimization Problems, Vol. 5015, Springer, pp. 278-282. 
Alon, N. and Edelman, P. H.: 2010, The inverse Banzhaf problem, Social Choice and Welfare 34, 371-377.

Alonso-Meijide, J. and Fiestras-Janeiro, M.: 2002, Modification of the Banzhaf value for games with a coalition structure, Annals of Operations Research 109, 213-227.

Aziz, H., Paterson, M. and Leech, D.: 2007, Efficient algorithm for designing weighted voting games. Working Paper of the University of Warwick.

Banzhaf, J. F.: 1965, Weighted voting does not work: a mathematical analysis, Rutgers Law Review 19, 317-343.

Bean, D., Friedman, J. and Parker, C.: 2008, Simple majority achievable hierarchies, Theory and Decision 65, 285-302.

Carreras, F. and Freixas, J.: 1996, Complete simple games, Mathematical Social Sciences 32, 139-155.

Carreras, F. and Freixas, J.: 2008, On ordinal equivalence of power measures given by regular semivalues, Mathematical Social Sciences 55, 221-234.

de Keijzer, B., Klos, T. and Zhang, Y.: 2010, Enumeration and exact design of weighted voting games, in W. van der Hoek, G. A. Kaminka, M. Luck and S. Sen (eds), Proc. of 9th Int. Conf. on Autonomous Agents and Multiagent Systems (AAMAS 2010), Cambridge University Press, pp. 391-398.

Deegan, J. and Packel, E. W.: 1978, A new index of power for simple n-person games, International Journal of Game Theory 7, 113-123.

Felsenthal, D. S. and Machover, M.: 1998, The measurement of voting power: Theory and practice, problems and paradoxes, Edward Elgar.

Felsenthal, D. S. and Machover, M.: 2004, A priori voting power: What is it all about?, Political Studies Review 2, 1-23.

Felsenthal, D. S., Machover, M. and Zwicker, W. S.: 1998, The bicameral postulates and indices of a priori voting power, Theory and Decision 44, 83-116.

Freixas, J. and Gambarelli, G.: 1997, Common internal properties among power indices, Control and Cybernetics 26, 591-604.

Freixas, J. and Marciniak, D.: 2009, A minimum dimensional class of simple games, TOP: An Official Journal of the Spanish Society of Statistics and Operations Research 17, 407-414.

Freixas, J., Marciniak, D. and Pons, M.: 2012, On the ordinal equivalence of the Johnston, Banzhaf and ShapleyShubik power indices, European Journal of Operational Research 216, 367-375.

Freixas, J. and Molinero, X.: 2009, On the existence of a minimum integer representation for weighted voting systems, Annals of Operations Research 166, 243-260.

Freixas, J. and Molinero, X.: 2010a, Detection of paradoxes of power indices for simple games, in L. A. Petrosjan and N. A. Zenkevich (eds), Contributions to Game Theory and Management, Graduate School of Management, Saint Petersburg State University, pp. 82-90.

Freixas, J. and Molinero, X.: 2010b, Weighted games without a unique minimal representation in integers, Optimization Methods and Software 25, 203-215.

Freixas, J. and Pons, M.: 2010, Hierarchies achievable in simple games, Theory and Decision 68, 393-404.

Friedman, J., McGrath, L. and Parker, C.: 2006, Achievable hierarchies in voting games, Theory and Decision 61, 305-318.

Holler, M. J.: 1982, Forming coalitions and measuring voting power, Political Studies 30, 262-271.

Isbell, J. R.: 1956, A class of majority games, Quarterly Journal of Mechanics and Applied Mathematics 7, 183187.

Johnston, R. J.: 1978, On the measurement of power: Some reactions to Laver, Environment and Planning A 10, 907-914.

Jung-Fa, T., Ming-Hua, L. and Yi-Chung, H.: 2008, Finding multiple solutions to general integer linear programs, European Journal of Operational Research 184, 802-809.

Kurz, S.: 2012a, On minimum sum representations for weighted voting games, Annals of Operations Research 196, 361-369.

Kurz, S.: 2012b, On the inverse power index problem, Optimization 61, 989-1011. 
Leech, D.: 2003, Power indices as an aid to institutional design: the generalised apportionment problem, in M. Holler, H. Kliemt, D. Schmidtchen and M. Streit (eds), Jahrbuch für Neue Politische Ökonomie, Vol. 22, Mohr Siebeck, pp. 107-121.

Leech, D. and Leech, R.: 2005, Voting power in the Bretton Woods institutions, Homo Oeconomicus 22, 1-23.

Owen, G.: 1977, Values of games with a priori unions, in R. Henn and O. Moeschlin (eds), Mathematical Economics and Game Theory, Springer, pp. 76-88.

Pulido, M. A. and Sánchez-Soriano, J.: 2009, On the core, the Weber set and convexity in games with a priori unions, European Journal of Operational Research 193, 468-475.

Shapley, L. S. and Shubik, M.: 1954, A method of evaluating the distribution of power in a committee system, American Political Science Review 48, 787-792.

Straffin, P. D.: 1980, Topics in the Theory of Voting, Boston:Birkhauser.

Strand, J. R.: 1999, State power in a multilateral context: Voting strength in the Asian Development Bank, International Interactions 25, 53-74.

Strand, J. R.: 2001, Institutional design and power relations in the African Development Bank, Journal of Asian and African Studies 36, 203-223.

Strand, J. R.: 2003, Measuring voting power in an international institution: the United States and the InterAmerican Development Bank, Economics of Governance 4, 19-36.

Taylor, A. D. and Pacelli, A. M.: 2008, Mathematics and politics: Strategy, voting, power, and proof, Springer.

Taylor, A. D. and Zwicker, W. S.: 1999, Simple games: Desirability relations, trading, and pseudoweightings, Princeton University Press, New Jersey, USA.

Telgen, J.: 1983, Identifying redundant constraints and implicit equalities in systems of linear constraints, Management Science 29, 1209-1222. 


\section{A A linear integer program for the WSVG: [51;49, 49, 2]}

Table A.1: Coalitions in WSVG: $[51 ; 49,49,2]$.

\begin{tabular}{ccccc}
\hline \hline Coalition & Voter 1 & Voter 2 & Voter 3 & Status \\
\hline 1 & 1 & 1 & 1 & Win \\
2 & 1 & 1 & 0 & Win \\
3 & 1 & 0 & 1 & Win \\
4 & 1 & 0 & 0 & Lose \\
5 & 0 & 1 & 1 & Win \\
6 & 0 & 1 & 0 & Lose \\
7 & 0 & 0 & 1 & Lose \\
8 & 0 & 0 & 0 & Lose \\
\hline \hline
\end{tabular}

1 denotes a Yes vote, 0 a No vote.

Table A.2: Constraints for WSVG: $[51 ; 49,49,2]$.

\begin{tabular}{lcccc}
\hline \hline 1 & $w_{1}+w_{2}$ & $>$ & 0 & \\
2 & $w_{1}+w_{3}$ & $>$ & 0 & \\
3 & $w_{2}+w_{3}$ & $>$ & 0 & \\
4 & $w_{1}+w_{2}+w_{3}$ & $>$ & 0 & \\
5 & $w_{2}$ & $>$ & 0 & $*$ \\
6 & $w_{3}$ & $>$ & 0 & $*$ \\
7 & $-w_{1}+w_{2}+w_{3}$ & $>$ & 0 & $*$ \\
8 & $w_{2}+w_{3}$ & $>$ & 0 & \\
9 & $w_{1}$ & $>$ & 0 & $*$ \\
10 & $w_{1}-w_{2}+w_{3}$ & $>$ & 0 & $*$ \\
11 & $w_{3}$ & $>$ & 0 & \\
12 & $w_{1}+w_{3}$ & $>$ & 0 & \\
13 & $w_{1}+w_{2}-w_{3}$ & $>$ & 0 & $*$ \\
14 & $w_{1}$ & $>$ & 0 & \\
15 & $w_{2}$ & $>$ & 0 & \\
16 & $w_{1}+w_{2}$ & $>$ & 0 & \\
17 & $w_{1}$ & $\geq$ & 0 & \\
18 & $w_{2}$ & $\geq$ & 0 & \\
19 & $w_{3}$ & $\geq$ & 0 & \\
\hline \hline
\end{tabular}




\section{B WSVGs with fewer than 5 voters}

Table B.1: List of raw indices for all WVSGs with $n \leq 4$ voters.

\begin{tabular}{|c|c|c|c|c|c|}
\hline$n \leq 4$ & $\mathcal{W}^{m}$ & $\overline{\text { WVG }}$ & MSR & Banzhaf & Shapley-Shubik \\
\hline (1)-a & 1 & {$[1 ; 1,0,0,0]$} & 1:0:0:0 & 8:0:0:0 & $24: 0: 0: 0$ \\
\hline \multirow[t]{2}{*}{ (2) } & $1 ; 2$ & {$[1 ; 1,1,0,0]$} & 1:1:0:0 & 4:4:0:0 & 12:12:0:0 \\
\hline & 12 & {$[2 ; 1,1,0,0]$} & - & - & - \\
\hline \multirow[t]{2}{*}{ (4) } & $1 ; 2 ; 3$ & {$[1 ; 1,1,1,0]$} & 1:1:1:0 & $2: 2: 2: 0$ & 8:8:8:0 \\
\hline & 123 & {$[3 ; 1,1,1,0]$} & - & - & - \\
\hline (6)-a & $12 ; 13 ; 23$ & {$[2 ; 1,1,1,0]$} & - & 4:4:4:0 & - \\
\hline \multirow[t]{2}{*}{ (7) } & $1 ; 2 ; 3 ; 4$ & {$[1 ; 1,1,1,1]$} & 1:1:1:1 & $1: 1: 1: 1$ & $6: 6: 6: 6$ \\
\hline & 1234 & {$[4 ; 1,1,1,1]$} & - & - & - \\
\hline \multirow[t]{2}{*}{ (9) } & $12 ; 13 ; 14 ; 23 ; 24 ; 34$ & {$[2 ; 1,1,1,1]$} & - & $3: 3: 3: 3$ & - \\
\hline & $123 ; 124 ; 134 ; 234$ & {$[3 ; 1,1,1,1]$} & - & - & - \\
\hline \multirow[t]{2}{*}{ (11) } & $12 ; 13$ & {$[3 ; 2,1,1,0]$} & $2: 1: 1: 0$ & $6: 2: 2: 0$ & $16: 4: 4: 0$ \\
\hline & $1 ; 23$ & {$[2 ; 2,1,1,0]$} & - & - & - \\
\hline \multirow[t]{2}{*}{ (13) } & $123 ; 124 ; 134$ & {$[4 ; 2,1,1,1]$} & $2: 1: 1: 1$ & $4: 2: 2: 2$ & $12: 4: 4: 4$ \\
\hline & $1 ; 23 ; 24 ; 34$ & {$[2 ; 2,1,1,1]$} & - & - & - \\
\hline$(15)-\mathrm{a}$ & $12 ; 13 ; 14 ; 234$ & {$[3 ; 2,1,1,1]$} & - & $6: 2: 2: 2$ & - \\
\hline \multirow[t]{2}{*}{$(16)$} & $12 ; 13 ; 14 ; 23 ; 24$ & {$[3 ; 2,2,1,1]$} & $2: 2: 1: 1$ & $4: 4: 2: 2$ & $8: 8: 4: 4$ \\
\hline & $12 ; 134 ; 234$ & {$[4 ; 2,2,1,1]$} & - & - & - \\
\hline \multirow[t]{2}{*}{ (18) } & $123 ; 124$ & {$[5 ; 2,2,1,1]$} & - & $3: 3: 1: 1$ & $10: 10: 2: 2$ \\
\hline & $1 ; 2 ; 34$ & {$[2 ; 2,2,1,1]$} & - & - & - \\
\hline \multirow[t]{2}{*}{ (20) } & $12 ; 13 ; 14$ & {$[4 ; 3,1,1,1]$} & 3:1:1:1 & $7: 1: 1: 1$ & $18: 2: 2: 2$ \\
\hline & $1 ; 234$ & {$[3 ; 3,1,1,1]$} & - & - & - \\
\hline \multirow[t]{2}{*}{$(22)$} & $1 ; 23 ; 24$ & {$[3 ; 3,2,1,1]$} & $3: 2: 1: 1$ & $5: 3: 1: 1$ & $14: 6: 2: 2$ \\
\hline & $12 ; 134$ & {$[5 ; 3,2,1,1]$} & - & - & - \\
\hline \multirow[t]{2}{*}{ (24) } & $12 ; 13 ; 234$ & {$[4 ; 3,2,2,1]$} & $3: 2: 2: 1$ & $5: 3: 3: 1$ & $10: 6: 6: 2$ \\
\hline & $12 ; 13 ; 234$ & {$[5 ; 3,2,2,1]$} & - & - & - \\
\hline
\end{tabular}

The adjective 'raw' refers to an index prior to normalization by the sum of voting weights. Non-numbered games have quota $q^{\prime}=w(N)-q+1$, where $q$ is the quota of the preceding game. If $q^{\prime}=q$, then the game is auto-dual. 